\title{
Mapping the recovery of critically ill COVID19 patients by high-dimensional profiling identifies longitudinal blood immunotypes
}

\author{
Penttilä PA \\ KULeuven \\ Van Gassen S \\ UGENT/VIB \\ Panovska D \\ KULeuven \\ Vanderbeke $\mathrm{L}$ \\ KULeuven/UZLeuven \\ Van Herck $Y$ \\ KULeuven/UZLeuven \\ Quintelier K \\ UGENT/VIB \\ Emmaneel A \\ UGENT/VIB
}

Filtjens $\mathrm{J}$

KULeuven

Malengier-Devlies B

KULeuven

Ahmadzadeh K

KULeuven

Van Mol P

KULeuven

Borras DM

KULeuven

Antoranz A

KULeuven

\section{Bosisio F}

KULeuven

Wauters E.

KULeuven/UZLeuven

Matthys $\mathbf{P}$ 
KULeuven

\section{Saeys $Y$}

UGENT/VIB

\section{Garg A}

KULeuven

\section{Wauters $\mathrm{J}$}

KULeuven/UZLeuven

De Smet F ( $\square$ frederik.desmet@kuleuven.be )

KULeuven https://orcid.org/0000-0002-6669-3335

\section{Research Article}

Keywords: COVID19, longitudinal sampling, blood profiling, intensive care, CYTOFn cytokines

Posted Date: November 25th, 2020

DOI: https://doi.org/10.21203/rs.3.rs-108268/v1

License: (c) (i) This work is licensed under a Creative Commons Attribution 4.0 International License. Read Full License 


\section{Abstract}

The COVID-19 pandemic poses a major burden on health-care and economic systems across the globe. Even though a majority of the population only develops minor symptoms upon SARS-CoV2 infection, a significant proportion are hospitalized at intensive care units (ICU) requiring critical care. While insights into the early stages of the disease are gradually expanding, the dynamic immunological processes occurring in critically ill patients throughout their recovery at ICU are far less understood. Here, we have analysed longitudinally collected, whole blood samples of 40 surviving COVID-19 patients during their recovery at ICU using high-dimensional cytometry by time-of-flight (CyTOF) and cytokine multiplexing. Based on the neutrophil to lymphocyte ratio (NLR), we defined 4 sequential immunotypes during recovery that correlated to various clinical parameters, including the level of respiratory support at concomitant sampling times. We also identified classical monocytes as the first immune cell type to recover by restoring HLA-DR-positivity and by reducing the immunosuppressive CD163+ monocyte population, followed by the recovery of $\mathrm{CD} 8+$ and $\mathrm{CD} 4+\mathrm{T}$ cell, and $\mathrm{mDC}$ populations. The identified immunotypes also correlated to aberrant cytokine and acute-phase reactant levels. Finally, integrative analysis of cytokines and immune cell profiles showed a shift from an initially dysregulated immune response to a more coordinated immunogenic interplay, highlighting the importance of longitudinal sampling to understand the pathophysiology underlying recovery from severe COVID-19.

\section{Introduction}

The SARS-CoV2 coronavirus outbreak has infected more than 40 million people worldwide causing more than 1 million deaths and was officially declared a pandemic by the World Health Organization (WHO) [1]. Due to the lack of immunity to SARS-CoV2 and in the absence of an effective vaccine, the unpredictable clinical outcome of COronaVirus-Induced Disease 2019 (COVID-19) has created significant strain on medical systems [2-5]. Until now, major research efforts have tried to unravel the pathobiology underlying the disease course [6, 7]. As such, it has been shown that COVID-19 leads to the combination of pathologically elevated levels of pro-inflammatory cytokines, coagulopathy and a dysregulated immune response $[6,8,9]$. Deep immune profiling in severe COVID-19 patients revealed excessive amounts of dysfunctional neutrophils, decreased levels of lymphocytes and low levels of antigenpresenting receptors on monocytes and dendritic cells, hindering efficient adaptive immune responses [8, 10-12]. In contrast to asymptomatic or mild/moderate symptomatic COVID-19 patients, critically ill patients typically show a biphasic disease course with an early viral stage of disease before ICU admission, followed by an hypoxic phase of disease, characterized by a cytokine storm and an acute respiratory distress syndrome, requiring (invasive) respiratory support, antivirals and immune-modulation to prevent multiple organ failure and death

Considering the potentially severe and unpredictable impact of a SARS-CoV2 infection, research has also focused on predicting the disease course of COVID-19. Using a variety of multiplexing technologies, 
longitudinal blood sampling revealed a dichotomous profile in cytokine expression levels that could early on differentiate patients prone to develop a mild/moderate versus a severe disease course within the first two weeks following SARS-CoV2 infection [13-15]. In addition, severely diverging levels of immune cell populations, typically reported by aberrant neutrophil-over-lymphocyte ratios (NLR) and high levels of immature neutrophil frequencies, were also indicative in the early stages of the disease for a more severe disease course [16-22]. Based on these insights, various interventional trials were initiated, from which the use of corticosteroids showed promising results [23] although the optimal timing of these interventions remains to be determined and side effects (i.e. secondary infections) may occur. Therefore, until an effective vaccine is identified, patients exhibiting severe pathology will continue to be admitted to ICU requiring extensive medical care.

In contrast to the early stages of the disease, the biology from ICU admission to the recovery phase remains far less understood. A systems-biology study including the longitudinal follow-up of 10 ICU patients, suggested involvement of eosinophils early in the disease course [24]. Various immune cell populations were also identified to be increased in relative proportions during recovery while excessive cytokine levels returned to normal levels at the time of discharge from ICU [24, 25] Also the restoration of $T$ cell functionality, including the induction of effector-memory cells, has been described during the recovery phase of COVID-19 [26]. However, the exact sequence of changes occurring during the restitution of the peripheral immune system after severe infection remains elusive.

Within the transdisciplinary clinical COntAGlouS trial (COvid-19 Advanced Genetic and Immunologic Sampling, NCT04327570), we have collected and analysed longitudinal whole blood and serum samples of critically ill COVID-19 patients that survived a stay at ICU at the University Hospitals Leuven, Belgium. By using high-dimensional analysis technologies, we identified 4 immunotypes (i.e. blood cell composition profiles) that were based on the NLR, defining a common biological trajectory during patient recovery which correlated well to various clinical parameters, including the required level of respiratory support at concomitant sampling times. We identified classical monocytes as the first cell type to recover, followed by CD8+ and CD4+ T cell, and MDC populations, while the NLR-based groups also correlated to cytokine and acute-phase reactant (APR) levels. Finally, integrative analysis of cytokines and immune cell profiles, allowed us to monitor the recovery from a dysregulated immune response, highlighting the importance of longitudinal sampling to better understand the pathophysiology underlying the recovery from severe COVID-19.

\section{Methods}

\section{Patient cohort and sampling}

Forty COVID-19 patients that were admitted to the ICU were enrolled in the Contagious trial (NCT04327570). Thirty eight patients were confirmed positive for SARS-CoV2 infection by nasopharyngeal swab and/or by bronchoalveolar lavage (BAL) sampling and subsequent PCR quantification. Two patients were confirmed positive for SARS-CoV2 via CT scan. Routine clinical 
laboratory tests at the time of sampling were performed in line with the 'COntAGlous' study design [12]. The patients enrolled in the study were sampled at several relevant time points: upon admission to the ICU (0-2 days post admission), at $6+/-2$ days post admission, and eventually at ICU discharge. Of note, if the medical condition of the patient was not improving, a bronchoscopy was performed during which whole blood samples were again collected for concomitant immune profiling.

The healthy control group consisted of volunteers (recruited from UZ Hospital staff) with no prior diagnosis or recent symptoms compatible with COVID-19. This group was not tested by nasopharyngeal swab, but antibody assessment of plasma samples revealed absence of COVID-19 IgG antibodies.

\section{Sample Processing and Staining Procedure for CyTOF}

Whole blood (WB) samples were collected into sterile anticoagulant lithium heparin blood collection tubes and processed for mass cytometry staining within 2-4 hours of isolation. WB was stained with the Maxpar ${ }^{\circledR}$ Direct $^{\text {tM }}$ Immune Profiling Assay (DIPA) kit from Fluidigm $\odot$ by following the workflow outlined for whole blood staining. Additional antibodies not included in the Maxpar DIPA kit were titrated on WB and RBC-free samples from donors to determine the optimal staining index. The antibodies are outlined in Suppl. Table 1. The additional markers were added after the red blood cell-removal step. The last step of the protocol is the 193-Ir intercalation, which was performed overnight at $4^{\circ} \mathrm{C}$. The samples that could not be acquired on the instrument the very next day were immediately cryopreserved in Maxpar Fix/Perm buffer +193 -Ir at $-80^{\circ} \mathrm{C}$. The cryopreservation technique was validated in triplicate by dividing aliquots of donor samples stained on the same day and comparing cell viability and immune profiles between the fresh and cryopreserved samples. Batch effects were evaluated by running a reference sample derived from an aliquot of the same healthy donor over the period of the study.

\section{Data Acquisition}

Cells stained for mass cytometry were acquired the day after the staining procedure or within 1 week of cryopreservation. For CyTOF acquisition, the samples were pelleted in Maxpar Cell Acquisition Buffer (CAS) on the day of acquisition and transferred to the KU Leuven Flow and Mass Cytometry Facility to be acquired on a Helios ${ }^{\circledR}$ mass cytometer (Fluidigm). Prior to acquisition, 1 million cells/ml were resuspended in CAS containing EQ beads $(1: 10)$ and filtered through $35 \mu \mathrm{m}$ cell strainer cap tubes. The samples were acquired at a rate of 250-300 events per second on a Helios ${ }^{\circledR}$ Mass Cytometer. CyTOF software version 6.7.1016 and the Maxpar Direct Immune Profiling Assay.tem template were used to acquire and normalize data from the stained samples.

\section{Chemokine and cytokine assays}

Chemokine and cytokine levels in plasma were assessed by Meso Scale Discovery using the V-plex human cytokine 30-plex kit, complemented with Human IL-1RA (V-plex) and human IL-18 (U-plex) kits.

\section{Data Analysis}


To analyse the data, we continued using the FlowSOM models built for [12]. To generate these models, normalized .fcs files were first transferred to the Maxpar Pathsetter ${ }^{\text {TM }}$ software (version 2.0.45), which performs a standardized, automatic and unsupervised quality check (bead removal and high-quality singlet selection) and gating of live single cells. Samples with fewer than 50000 cells were discarded, as were 4 samples which showed up as outliers in a PCA analysis of the 25, 50 and 75 percent quantiles of the marker values. The FlowSOM analysis was subsequently done in $\mathrm{R}$, using 123 cleaned .fcs files, including 8 healthy controls and longitudinal samples of 40 COVID-19 patients, through various stages of the disease. Samples were first pre-processed: margin events were filtered out, data was transformed with an arcsinh transformation with cofactor 5 and the PeacoQC algorithm (manuscript in preparation, tool available at https://github.com/saeyslab/peacoQC) was applied to remove any unstable signal regions during the measurement. On this cleaned data a first FlowSOM model [27] was trained, using a random selection of cells for all samples, resulting in 3,000,093 cells to train on. The clustering made use of 11 markers (CD3, CD4, CD8a, CD11c, CD14, CD19, CD20, CD45, CD66b, TCR $\$ O and NCAM), mapped the data onto a 10 by 10 SOM grid and resulted in 30 meta-clusters. 22 meta-clusters were selected as having CD66b values lower than 2 or CD45 values higher than 4, corresponding to non-granulocytes, while 8 meta-clusters were labelled as granulocytes. The full files were mapped onto this model, and for each of them new fcs files were generated corresponding to the two subsets of cells. These were then used to build two separate FlowSOM models, including either the non-granulocyte or the granulocyte cells, again using only a subset of 2,949,946 (granulocyte: $3,000,093$ ) cells for training mapped onto a 10 by 10 SOM grid, this time using 33 markers (CD3, CD4, CD8a, CD11c, CD14, CD16, CD19, CD20, CD27, CD28, CD38, CD45, CD45RA, CD45R0, CD57, CD66b, CD69, CD294, CD161, CD163, CCR4, CCR6, CCR7, CXCR3, CXCR5, HLA-DR, IgD, IL-2Ra, IL-3R, IL-7Ra, NCAM, NKG2A and TCR囚ठ). To be able to identify small populations, no meta-clustering was applied on these second models, and the 100 clusters were manually annotated by 3 independent experts according to their mean fluorescence intensity (MFI) values. In the non-granulocyte model, 6 clusters were manually identified as still being mixtures of different cell types, and split into 2 or 3 clusters, resulting in 107 non-granulocyte clusters and 100 granulocyte clusters.

For this study, all samples were again fully mapped onto these models to identify their immune profiles. During this process, we noticed some files had a sub-optimal granulocyte / non-granulocyte split. Therefore, we redefined this split using the file specific MFI values of the metaclusters, selecting all metaclusters with CD66b values lower than 3 and CD 45 values higher than 3 as non-granulocytes. For one file, 3 metaclusters were still wrongly assigned when inspecting the density distribution of the cells, and for this file the CD45 boundary was adapted to 4 . Once all cells were assigned to the 207 clusters from the existing models, we used a manual annotation of these clusters to aggregate them in 24 cell populations, describing the immune profile of each sample by the percentage of live cells each of these populations represented. Additionally, the total lymphocyte percentage (i.e. the sum of all populations which were annotated as lymphocyte subsets) was added, bringing the total to 25 parameters per sample. The samples were grouped in 4 equal sized groups depending on their NLR: 31 samples with a ratio higher than 7.1 (R4), 30 samples with a ratio between 7.1 and 4.8 (R3), 31 samples with a ratio between 4.8 and 2.7 (R2) and 31 samples with a ratio lower than 2.7 (R1). The four profiles were ordered 
by decreasing neutrophil/lymphocyte ratio, and are throughout the figures in this paper colored as black, green, blue and cyan respectively.

The differences between these four groups were then further characterized. To compute statistics, we made use of linear mixed models, and for the cytokines and ARP values we used mixed models with random effect for the patient, to correct for the fact that patients can have multiple longitudinal samples, spread over one or more groups. Correlations were calculated using the Spearman Rank test. All p-values were taken together to be corrected for multiple testing with the Benjamini-Hochberg (FDR) method. Curve fitting analysis was done using the drc package in $\mathrm{R}$ from which the inflection points and the corresponding $p$-values were collected. Additionally, we also used the built-in immuno profiling tools of the Maxpar Pathsetter ${ }^{\text {TM }}$ software to analyse the overall immune cell profile in a standardized and automated way. The Pathsetter-based blood profiles were grouped into 12 clusters using k-means clustering, and manually further grouped into 4 final profiles. These profiles strongly corresponded with the NLR results and led to similar biological conclusions, thus giving additional trust in the results.

The code used to generate the results is available at https://github.com/saeyslab/CYTOF_covid19_study.

To create the correlation matrices, we applied the Morpheus software (https://software.broadinstitute.org/morpheus) and used Pearson correlation metric for clustering. For clustering, only those values were considered for COVID-19 patients that were matched across both serum cytokine/chemokine screening as well as mass cytometry. Unmatched patients (if any) were excluded.

\section{Results}

\section{Mapping the disease course of COVID-19 survivors at ICU}

To unravel the sequential recovery of the immune system throughout the course of a severe COVID-19 infection, we have analysed a cohort of 40 patients that survived a stay at the intensive care unit (ICU) at the University Hospitals Leuven, Belgium. These patients were enrolled in the COntAGlouS trial (NCT04327570) through which longitudinal whole blood samples were collected during their ICU stay (see methods and Table 1 for patient demographics and characteristics; a set of healthy controls was included for reference).

For each patient, a detailed timeline of their ICU stay was generated displaying the most clinically relevant events including onset of symptoms, hospital admission, ICU admission, start/stop of therapeutic interventions and finally discharge from ICU and hospital. If applicable, discharge from a specialized revalidation centre where the patient continued his/her recovery after their hospital stay, is also included. Occasionally, blood was drawn at additional time points (such as during bronchoscopy), which is indicated on the timeline. Once individual time points were plotted (Figure 1A), a large range in the duration of ICU stay (from 2 to 72 days) was found, indicating a highly variable recovery rate across 
patients. From the 40 enrolled patients, only 9 were women, corresponding to previous observations of males experiencing increased severity and hospitalization rates once infected with COVID-19 [28-30] (Figure 1B). The age range and overall time spent at ICU of the admitted patients was however comparable for men and women (Figure 1B and Table 1). Within this critically ill patient cohort, we did not find a correlation between BMI and the overall duration of ICU stay $(r=-0.15, p=0.35)$, despite $>40 \%$ of the patients having a BMI above 30 (Table 1, Figure 1B) [31].

This timeline was further extended with the level of respiratory support that was required at concomitant sampling time points (Figure 1C, D). Here, respiratory support levels were classified from 0-5 where level 0 indicates no support, while level 4 and 5 indicate the need for mechanical ventilation (with level 5 indicating patients that were ventilated in prone position and/or requiring inhalation NO therapy or extracorporeal membrane oxygenation (ECMO)) (Table 1). Subsequent correlation analysis showed that the maximal level of respiratory support correlated well to the overall duration of ICU stay $(r=0.67, p=7 e-$ 17, Figure 1C).

\section{Identifying peripheral blood profiles using longitudinal sampling}

Next, we performed deep immune profiling on collected blood samples using high dimensional cytometry by time-of-flight (CyTOF) analysis. From this data, 33 surface markers were used to map changes in composition and phenotype of white blood cells during ICU stay. All data were mapped using unsupervised gating methods and manually annotated to identify the immune cell subtypes to reconstruct potential dynamic changes. However, given the variation in duration of hospitalization at the ICU in this patient cohort, mapping immune profiles on an absolute time scale did not produce coherent results. Alternatively, we ranked samples based on the neutrophil-to-lymphocyte ratio (NLR) and defined 4 equal groups (Figure 2A-C) across all collected samples, regardless of when a sample was taken. As such, we defined 4 "NLR" stages (R1-4) ranging from severe lymphopenia and neutrophilia (profile R4, which showed the highest NLR values) to immune profiles resembling those of healthy controls, defined by the lowest NLR values (R1) (Figure 2A, B and Suppl. Figure 1). In addition, a high NLR (R4) also correlated to increased levels of the acute phase reactants C-reactive protein (CRP) and ferritin (Figure 2C, D), markers that are commonly monitored in ICU patients and define a critical state.

\section{Integrating longitudinal immune-profiling with clinical features}

Next, we assessed how the identified NLR groups correlated to a variety of clinical parameters. As indicated above, the level of respiratory support was measured at each sampling time point and correlation analysis of this feature with the 4 NLR profiles revealed that patients exhibiting R4 required increased respiratory support at that moment compared to lower level NLRs (Figure 2E). Further correlation analysis also showed that the overall clinical status of each patient upon admission to ICU, as determined by calculating the Sequential Organ Failure Assessment Score (SOFA) [32, 33], was significantly correlated to the NLR groups (Figure 2F; $r=-0.23, p=0.03$ )[33]. We did not find any correlations of age or BMI with the NLR groups (Suppl. Figure 3A, B). 
Using these groups, we reconstructed a detailed sequence of events for each individual patient and investigated how these evolved along their ICU stay (Figure 1E, 2G, Suppl. Figure 4). For 36 out of 40 patients, we observed a recovery (overall downwards shift of the NLR score) or stabilisation of the immune system (and NLR score) along their stay at ICU (Suppl. Figure 4), with an enrichment of R1 towards ICU discharge (Figure 2G). Strikingly, the R values at ICU discharge still ranged between R1-4 suggesting that the physical condition of these patients was still heterogeneous at that moment. In line with this observation, we found that patients exhibiting a higher NLR value upon discharge from ICU required significantly longer revalidation (either at the regular hospital ward and/or a specialized revalidation center) following their stay at ICU (Figure $2 \mathrm{H}, \mathrm{r}=-0.4, \mathrm{p}=0.02$ ). Additionally, Charlson comorbidity indices [34] were low for all patients (Table 1), indicating that the majority of patients admitted to ICU were in good general health before their infection with SARS-CoV2. We also did not observe correlations of the Charlson comorbidity indices to either the NLRs or the required level of respiratory support (Suppl. Figure 3C, D).

Finally, we tracked alterations in patient's immune profiles who were treated with diverse immunomodulatory regimens. Commonly, the glucocorticoid methylprednisolone (MP) was given as a monotherapy $(n=19)$. In some cases, MP was prescribed in a combination with either anti-IL-1 (anakinra; $\mathrm{n}=3$ ) or anti-IL-6 (tocilizumab; $\mathrm{n}=5$ ). One patient received anti-IL-6 exclusively, while the remaining 12 patients in our cohort relied on supportive care alone without immunomodulatory treatments (Table 1; Figure 1F). Unsurprisingly, our data analysis confirmed that patients who underwent steroid treatment, had a greater tendency towards a longer ICU stay. Correlation analysis of the NLR profiles with MP treatment, revealed that patients receiving MP exhibited a higher NLR upon ICU discharge, as opposed to patients that did not receive MP (Suppl. Figure 3E). Finally, while the cohort of patients that received antiIL-1 and anti-IL- 6 were small, the reconstitutional trajectory of their immune systems followed a similar pattern to those patients receiving standard-of-care treatment without immunomodulatory drugs and/or MP.

\section{Reconstructing the cellular recovery of critically ill COVID-19 patients}

Considering that the NLR levels correlated well with the clinical condition of the patients (see above), we next investigated the recovery of more specific immune cell populations to eventually define a sequence of events. To do so, we used a dual approach: first, we performed curve fitting along the trajectory of the four NLR profiles, in which we determined the inflection points of each identified immune cell population to estimate the moment recovery would begin (Figure 3A, B, Suppl. Table 2). The data from this approach were subsequently combined with the statistical comparison of the 4 NLR groups (Figure 2A, suppl. Table 3 ) to define the eventual order by which specific cell populations were recovering. We found that, on average across this cohort of patients, classical monocytes were the first immune cell population to recover followed by naive CD8+ T cells, naive and Th2-polarized CD4+ T cells. As indicated by our analysis, effector and memory $T$ cell populations were only restored at later stages, including the antiviral Th1-polarized CD4+ T cells [35]. From the other professional antigen presenting cells, myeloid dendritic cells $(\mathrm{mDC})$ were recovering immediately after the early $T$ cell response, while plasmacytoid DCs 
(pDC) would only recover towards R1, similar to overall B and NK cell populations. This suggests that monocytes, as implied by other studies, could become important targets in both understanding COVID-19 disease progression, as well as, improving recovery at the early phase of ICU admission [36].

\section{Functional recovery of monocytes}

Antigen-presentation by monocytes, pDCs and/or mDCs plays a vital role in the initiation of an efficient adaptive immune response to viral infections [37-41]. As previously shown, declined levels of HLA-DR expression and hence a reduced antigen presentation seems to be an early hallmark of a trajectory towards a severe COVID-19 disease course compared to a mild/moderate one [10,12]. Considering that monocytes are among the first immune cell types to recover, we further investigated the functionality of these cells across the 4 NLR profiles during recovery. As such, we observed a regain in both the numbers and the antigen presenting capabilities of the monocytes towards discharge (figure 3C). The first subset to re-establish were the classical HLA-DR+ monocytes (Cluster (Cl)3, $\mathrm{Cl} 33$ and $\mathrm{Cl} 12$; figure 3C), while it was only in later stages of disease progression that the non-classical HLA-DR+ monocytes were restored (see $\mathrm{Cl} 11$, Figure 3C; see also statistics in Figure 2A).

The early phase of severe COVID19 is characterized by a functional shift towards a more immunosuppressive spectrum of monocytes, as seen by a downregulation of HLA-DR and an enrichment of CD163+ monocytes in R4 (Figure 3) [10,12]. However, longitudinal follow-up shows that this shift is reversible, as seen by the recovery of HLA-DR expression and a relative reduction of the amount of immunosuppressive $\mathrm{CD} 163+$ monocytes ( see $\mathrm{Cl} 53$ and $\mathrm{Cl} 32$; Figure $3 \mathrm{C}$ ), leading to a regain of the antigen-presenting phenotype of these cells.

\section{Longitudinal cytokines profiling}

Next to immunophenotyping, we also performed multiplexed analysis of soluble serum proteins, which included 32 pro-inflammatory cyto- and chemokines in serum samples at concomitant sampling times. The levels of these analytes were subsequently correlated to the NLR profiles. In line with previous

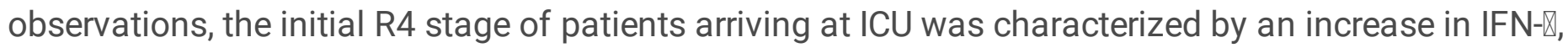
TNF-a, IL-2, IL-6, IL-7, IL-10, IL-15, IP-10, MCP-1, MIP-1 a, MIP-1 $\beta$ levels and a reduction in TARC, MDC and IL1- $a$, suggestive of a pro-inflammatory cytokine signature, often referred to as a "cytokine storm" (Figure 4A). This signature was steadily reversed as patients attained a normal R1 stage. Similarly, CRP and ferritin were also significantly higher in R4 and gradually decreased as patients reached R1 suggestive of a return to baseline following an acute phase induction of the immune system (Figure 2D,E). Clustering analysis to uncover patterns in cytokine profiles across the various NLR groups further confirmed that pro-inflammatory cytokine levels collectively reduced along the duration of an ICU stay (Figure 4B). On the other hand, MDC and TARC, two constitutive chemokines designated as CCL17 and CCL22 that are regulated at a post-translational level, increased to normal levels [42].

Even though the levels of cytokines within the R1 profile remained comparable between COVID-19 patients and healthy individuals, we observed altered expression levels of TNF-a, IL-18, IL1-a, MCP-1 and 
MIP-1a, between the two study groups(Figure 4A; healthy controls are indicated as hollow black circles in the figure). It remains to be studied how long it would take for levels to normalise, however, in spite of their aberrant profiles, patients were still able to leave the ICU for their recovery.

\section{Integrative mapping of the immune response during recovery}

Considering the important interplay between immune cells, cytokines and chemokines during severe COVID-19, we also performed an integrative, similarity matrix-based correlative statistical modelling analyses (Figure 5), to uncover associative patterns along the duration of a patient's recovery at ICU. As recently determined, critically ill COVID-19 patients are characterized by a strong dysregulation of the immune reaction against the SARS-CoV2 virus, whereby normally highly concerted interplays of cyto/chemokines and specific immune cell populations are now disentangled [8]. In line with above observations, our similarity matrix analyses also revealed an intense correlation between various immune cells and cyto/chemokines (a putative indication of ongoing immunological interactions) in the R4 critically-ill COVID-19 patients (Figure 5A), such that these correlations progressively "normalized" or decreased in terms of number of intense clusters when traversing from R4 to R1 (Figure 5A-D), thereby indicating that apart from above quantitative shifts, there was also a qualitative shift in possible immuneinteractions when going from critically-ill to recovering COVID-19 patients.

Furthermore, the immunological characteristics of these clusters within the four blood profiles were of particular interest considering the importance of both neutrophils and lymphocytes in marking the health status of COVID-19 patients. Interestingly, neutrophils exhibited proficient correlation with mainly adaptive immunity-relevant cytokines (e.g., IFN-X, IP-10/CXCL10, IL-2, IL-13, IL-15), pro-neutrophilic IL-8 and CD163+ intermediate-monocytes in R4 (Figure 5A). However, in R3, apart from the above-mentioned cytokines, neutrophils gained correlation with TNF-a, IL-10, MCP-1/CCL2, $\varangle \delta T$ cells, IL-17 and effector/naive CD4 T cells, a sign of slightly better (albeit pro-inflammatory) cross-talk with adaptive immune cells and some degree of immunoregulation (IL-10/IL-17) (Figure 5B). However, the neutrophilcentred clusters considerably "contracted" in R2/R1 (Figure 5C-D); ultimately resulting in a more neutrophil-specific immune-cluster consisting of the pro-neutrophilic chemokine (IL-8) and inflammasome footprint (IL-1 $\beta / I L-1 R a)$, a potent sign of anti-pathogenic neutrophil activity [43].

On the level of lymphocytes, the most striking phenotypes were observed in the R4 profile (Figure 5A) whereas patterns in R3/R2 were largely transitional and ultimately culminated into "contracted" lymphocytic correlative-compartment in R1 (Figure 5B-D). More specifically, in R4 patients (Figure 5A), the typically infection-resolving lymphocytic compartment (e.g. Th1/Th2 cells, effector/memory CD4/CD8 T cells) had relatively less correlations with various effector-function cytokines including IFN- $\mathbb{\text { , TNF- }}$, IL-2, IL-15, thereby indicating a certain degree of immunological dysregulation. Most of these lymphocytes also had considerable negative correlation with neutrophils. Interestingly TNF- $a$, Th17 and Tregs formed a cluster together which might be a sign of pro-inflammatory signalling since Tregs and Th17 have been shown to reciprocally stimulate each other via TNF-signalling pathway [44]. We believe that in the current context, this crosstalk might play a disease-potentiating role in COVID-19 severity. These discords were 
largely ameliorated from R3 to R1 (e.g., Th17 gaining correlation with Th1/Th2/Tfh and activated-CD8 T cells in R1) thereby indicating better lymphocytic activity/regulation to be beneficial for recovery of COVID-19 patients.

In conclusion, above qualitative analyses revealed that neutrophil/lymphocyte-associated inflammation undergoes considerable changes in terms of co-associative immune-components between R4 to R1 such that a relatively contracted neutrophil cluster with a chemotactic/inflammasome-footprint and a contracted lymphocyte cluster defines favourable recovery for COVID-19 patients.

\section{Discussion}

We have performed a longitudinal study in which consecutive peripheral blood samples of 40 surviving COVID-19 patients were analysed via high-dimensional mass cytometry analysis, allowing us to uncover detailed insights in the amount and functionality of the white blood cell (WBC) compartment. Importantly, by using fresh, non-cryopreserved whole blood samples, our study allowed the analysis of both granulocytes, from which neutrophils take the mainstay and play an important role in COVID-19 [45], in addition to the most important populations of lymphocytes.

Using those insights, we grouped all measured samples based on the NLR, as such identifying 4 blood profiles that defined a biological trajectory through which patients evolved at a highly individualized pace during recovery. Importantly, these NLR profiles correlated well to various clinical variables, including the level of respiratory support needed at concomitant sampling times. Another striking observation was the large variety of NLR levels upon discharge from ICU, which indicates that patients upon discharge from ICU, still exhibit a broad range of physical fitness, as suggested by the need for a longer revalidation following discharge from ICU when an increased NLR was measured.

Next to general clinical features, this cohort also included patients that received various therapeutic interventions, from which the use of corticosteroids (CS) was the most prominent. Our analysis showed that the likelihood of patients receiving CS therapy, correlated well with the duration of ICU stay, indicative of a good clinical selection of patients for this intervention. In addition, correlation analysis of the NLR profiles between patient groups that did and did not receive CS therapy, suggests that while both groups exhibited similar trajectories from high to lower ratio levels, the CS group showed a slower evolution towards a normal ratio. While it is known that CS therapy dampens the overall immune response [46], the excessive levels of cytokines and concurrent defective immune response as observed in COVID-19, warrants treatment with CS, in line with other studies [23]. Due to the small number of patients receiving anti-IL1 or anti-IL6, we cannot make conclusions towards changes in the recovery patterns of their peripheral immune system, even though we did not observe differences with those patients that did not receive these treatments. Overall, the timing of these therapeutic interventions remains critical and still requires further optimization $[47,48]$. Indeed, one of the major problems seems to be the delay of the innate immune system to efficiently respond to SARS-CoV2 infection, which further delays the subsequent adaptive immune response. While this cohort is small, it would be useful to track the 
phenotypes of severe "long-stay" vs "short-stay" patients as well as non-hospitalized COVID "Iong haulers" to identify early biomarkers in order to better predict immune responses to the virus [49].

The defined biological trajectory also allowed us to further elucidate the sequence of events and study the order in which particular immune cell populations reappear in the peripheral blood including the analysis of certain functionalities. Using this approach, we identified classical monocytes to recover first, followed by CD8+ and CD4+ T cells. While these observations are in line with other smaller studies [25, 36], they will need further corroboration in larger cohorts. Overall, the underlying biology suggests that protecting and/or reactivating the innate immune system (from which the monocytes seem first to recover), could be a potential therapeutic strategy. Indeed, based on these fundamental insights, therapies aimed at improving early monocyte functionality could be an interesting venue to improve the disease course, as suggested by the role for MCP1/CCR2 interference therapy in SARS-CoV1 [12, 50]_but also in COVID-19 as previously suggested [36]. We should however also look beyond monocytes: a reactivation of the other members of the innate immune system, including DCs and NK cells, could have the potential to ignite a stronger adaptive immune response and recovery. It also remains to be seen how specific the observed patterns are for COVID-19 and how they diverge from other severe respiratory diseases to tailor future interventions. Moreover, the absence of a comparator cohort on non-survivors could make these observations stronger.

Finally, next to the immune cell profiles, we also assessed concurrent levels of various cytokines and chemokines and acute phase reactants (i.e. CRP and ferritin). Clustering analysis revealed the gradual release of the pathologically elevated cytokine and chemokine levels, often referred to as a "cytokine storm", even though the levels in COVID-19 patients are still lower compared to some auto-inflammatory disorders e.g. macrophage activation syndrome. When patients evolved toward lower NLR levels, the coordination of the immune system recovered with a better alignment of cytokines and inflammatory cell types. Indeed, qualitative, correlative matrix analyses further revealed that hyper-inflammatory neutrophil clusters and dysregulated lymphocyte clusters distinguished critically-ill COVID-19 patients. Accordingly, amelioration of these features (i.e., contraction of neutrophil-clusters down to neutrophil-specific immunesignalling and consolidation of lymphocyte clusters to better crosstalk between different $\mathrm{T}$ cell subsets) marked the recovery phase in COVID-19.

\section{Declarations}

Ethics: This study was approved by the medical ethical committee of UZLeuven/KULeuven (study number S63881) and is also registered here: https://clinicaltrials.gov/ct2/show/NCT04327570

Competing Interests: The authors declare no competing interests.

\section{Acknowledgements}

We would like to thank Tatjana Verbeke, Marleen Derweduwe, Annelies Claeys, Reena Chinnaraj and Jenny Sprooten for technical support for the CyTOF experiments. This work was supported by FWO grant 
I007418N, KULeuven grant C14/17/084, KOOR funding and 'het Leuven Kankerinstituut'. S.V.G. is an ISAC Marylou Ingram Scholar and supported by an FWO postdoctoral research grant (Research Foundation Flanders). L. V. is supported by an FWO PhD fellowship (Research Foundation - Flanders, E11E9819N).

\section{Author contributions:}

Conceptualization, F.DS; Methodology, PA.P., S.VG., D.P.; Software/Data Analysis, S.VG., K.Q, A.E., A.A.M., Y.S., F.B., F.DS.; Investigation, PA.P, F.DS.; Clinical Trial Design, Data Acquisition and Interpretation, L.V., J.W., Y.VH., E.W., P.VM.; Cyto/Chemokine Data Acquisition, J.F, B.M.D., K.A.; Integrative Mapping and Interpretation of Cyto/Chemokine data, AD.G., DM.B.; Writing-Original Draft, PA.P., S.VG., D.P., AD.G., F.DS.; Writing-Review \& Editing, PA.P., S.VG., D.P., L.V., Y.VH., P.VM., E.W., P.M., AD.G., J.W., and F.DS. This work was fully supported by the Contagious Consortium.

\section{References}

1. Update WE, Asia S, Asia S (2020) Coronavirus disease ( COVID-19)

2. Ma X, Vervoort D (2020) Critical care capacity during the COVID-19 pandemic: Global availability of intensive care beds. J Crit Care 58:96-97. https://doi.org/10.1016/j.jcrc.2020.04.012

3. Ma X, Vervoort D, Reddy CL, et al (2020) Emergency and essential surgical healthcare services during COVID-19 in low- and middle-income countries: A perspective. Int J Surg 79:43-46. https://doi.org/10.1016/j.ijsu.2020.05.037

4. Bong C-L, Brasher C, Chikumba E, et al (2020) The COVID-19 Pandemic: Effects on Low- and MiddleIncome Countries. Anesth Analg 131:86-92. https://doi.org/10.1213/ANE.0000000000004846

5. Bielicki JA, Duval X, Gobat N, et al (2020) Monitoring approaches for health-care workers during the COVID-19 pandemic. Lancet Infect Dis. https://doi.org/10.1016/S1473-3099(20)30458-8

6. Blanco-Melo D, Nilsson-Payant BE, Liu W-C, et al (2020) Imbalanced Host Response to SARS-CoV-2 Drives Development of COVID-19. Cell 181:1036-1045.e9. https://doi.org/10.1016/j.cell.2020.04.026

7. Mann ER, Menon M, Knight SB, et al (2020) Longitudinal immune profiling reveals distinct features of COVID-19 pathogenesis. medRxiv 2020.06.13.20127605. https://doi.org/10.1101/2020.06.13.20127605

8. Wauters E, Thevissen K, Wouters C, et al (2020) Establishing a Unified COVID-19 "Immunome": Integrating Coronavirus Pathogenesis and Host Immunopathology. Front Immunol 11:1642. https://doi.org/10.3389/fimmu.2020.01642

9. Pedersen SF, Ho Y-C (2020) SARS-CoV-2: a storm is raging. J Clin Invest 130:2202-2205. https://doi.org/10.1172/JCl137647

10. Schulte-Schrepping J, Reusch N, Paclik D, et al (2020) Severe COVID-19 Is Marked by a Dysregulated Myeloid Cell Compartment. Cell. https://doi.org/10.1016/j.cell.2020.08.001

11. Wilk AJ, Rustagi A, Zhao NQ, et al (2020) A single-cell atlas of the peripheral immune response in patients with severe COVID-19. Nat Med 26:1070-1076. https://doi.org/10.1038/s41591-020-0944-y 
12. Vanderbeke L, Van Mol P, Van Herck Y, et al (2020) Monocyte-Driven Atypical Cytokine Storm and Aberrant Neutrophil Activation as Key Mediators of COVID19 Disease Severity. Ssrn

13. Lucas C, Wong $P$, Klein J, et al (2020) Longitudinal analyses reveal immunological misfiring in severe COVID-19. Nature 584:463-469. https://doi.org/10.1038/s41586-020-2588-y

14. Zhao Y, Qin L, Zhang P, et al (2020) Longitudinal COVID-19 profiling associates IL-1RA and IL-10 with disease severity and RANTES with mild disease. JCl insight 5:.

https://doi.org/10.1172/jci.insight.139834

15. Del Valle DM, Kim-schulze S, Hsin-hui H, et al (2020) An inflammatory cytokine signature helps predict COVID-19 severity and death. medRxiv 2020.05.28.20115758.

https://doi.org/10.1101/2020.05.28.20115758

16. Liu J, Liu Y, Xiang P, et al (2020) Neutrophil-to-lymphocyte ratio predicts critical illness patients with 2019 coronavirus disease in the early stage. J Transl Med 18:206. https://doi.org/10.1186/s12967020-02374-0

17. Fu J, Kong J, Wang W, et al (2020) The clinical implication of dynamic neutrophil to lymphocyte ratio and D-dimer in COVID-19: A retrospective study in Suzhou China. Thromb Res 192:3-8. https://doi.org/10.1016/j.thromres.2020.05.006

18. Yang A-P, Liu J-P, Tao W-Q, Li H-M (2020) The diagnostic and predictive role of NLR, d-NLR and PLR in COVID-19 patients. Int Immunopharmacol 84:106504. https://doi.org/10.1016/j.intimp.2020.106504

19. Ma A, Cheng J, Yang J, et al (2020) Neutrophil-to-lymphocyte ratio as a predictive biomarker for moderate-severe ARDS in severe COVID-19 patients. Crit Care 24:288. https://doi.org/10.1186/s13054-020-03007-0

20. Liu J, Li S, Liu J, et al (2020) Longitudinal characteristics of lymphocyte responses and cytokine profiles in the peripheral blood of SARS-CoV-2 infected patients. EBioMedicine 55:102763. https://doi.org/10.1016/j.ebiom.2020.102763

21. Carissimo G, Xu W, Kwok I, et al (2020) Whole blood immunophenotyping uncovers immature neutrophil-to-VD2 T-cell ratio as an early marker for severe COVID-19. Nat Commun 11:5243. https://doi.org/10.1038/s41467-020-19080-6

22. Silvin A, Chapuis N, Dunsmore G, et al (2020) Elevated Calprotectin and Abnormal Myeloid Cell Subsets Discriminate Severe from Mild COVID-19. Cell 182:1401-1418.e18. https://doi.org/10.1016/j.cell.2020.08.002

23. RECOVERY Collaborative Group, Horby P, Lim WS, et al (2020) Dexamethasone in Hospitalized Patients with Covid-19 - Preliminary Report. N Engl J Med. https://doi.org/10.1056/NEJMoa2021436

24. Rodriguez L, Pekkarinen PT, Lakshmikanth T, et al (2020) Systems-Level Immunomonitoring from Acute to Recovery Phase of Severe COVID-19. Cell reports Med 1:100078. https://doi.org/10.1016/j.xcrm.2020.100078

25. Wen W, Su W, Tang H, et al (2020) Immune cell profiling of COVID-19 patients in the recovery stage by single-cell sequencing. Cell Discov 6:31. https://doi.org/10.1038/s41421-020-0168-9 
26. Odak I, Barros-Martins J, Bošnjak B, et al (2020) Reappearance of effector T cells is associated with recovery from COVID-19. EBioMedicine 57:102885. https://doi.org/10.1016/j.ebiom.2020.102885

27. Van Gassen S, Callebaut B, Van Helden MJ, et al (2015) FlowSOM: Using self-organizing maps for visualization and interpretation of cytometry data. Cytometry A 87:636-45. https://doi.org/10.1002/cyto.a.22625

28. Jin J-M, Bai P, He W, et al (2020) Gender Differences in Patients With COVID-19: Focus on Severity and Mortality. Front public Heal 8:152. https://doi.org/10.3389/fpubh.2020.00152

29. Chen $\mathrm{T}$, Wu D, Chen $\mathrm{H}$, et al (2020) Clinical characteristics of 113 deceased patients with coronavirus disease 2019: retrospective study. BMJ 368:m1091. https://doi.org/10.1136/bmj.m1091

30. Gemmati D, Bramanti B, Serino ML, et al (2020) COVID-19 and Individual Genetic Susceptibility/Receptivity: Role of ACE1/ACE2 Genes, Immunity, Inflammation and Coagulation. Might the Double X-chromosome in Females Be Protective against SARS-CoV-2 Compared to the Single X-Chromosome in Males? Int J Mol Sci 21:. https://doi.org/10.3390/ijms21103474

31. Rod JE, Oviedo-Trespalacios O, Cortes-Ramirez J (2020) A brief-review of the risk factors for covid-19 severity. Rev Saude Publica 54:60. https://doi.org/10.11606/s1518-8787.2020054002481

32. Vincent JL, de Mendonça A, Cantraine F, et al (1998) Use of the SOFA score to assess the incidence of organ dysfunction/failure in intensive care units: results of a multicenter, prospective study. Working group on "sepsis-related problems" of the European Society of Intensive Care Medicine. Crit Care Med 26:1793-800. https://doi.org/10.1097/00003246-199811000-00016

33. Singer M, Deutschman CS, Seymour CW, et al (2016) The Third International Consensus Definitions for Sepsis and Septic Shock (Sepsis-3). JAMA 315:801-10. https://doi.org/10.1001/jama.2016.0287

34. Charlson ME, Pompei P, Ales KL, MacKenzie CR (1987) A new method of classifying prognostic comorbidity in longitudinal studies: development and validation. J Chronic Dis 40:373-83. https://doi.org/10.1016/0021-9681(87)90171-8

35. Maloy KJ, Burkhart C, Junt TM, et al (2000) CD4(+) T cell subsets during virus infection. Protective capacity depends on effector cytokine secretion and on migratory capability. J Exp Med 191:215970. https://doi.org/10.1084/jem.191.12.2159

36. Chua RL, Lukassen S, Trump S, et al (2020) COVID-19 severity correlates with airway epitheliumimmune cell interactions identified by single-cell analysis. Nat Biotechnol 38:970-979. https://doi.org/10.1038/s41587-020-0602-4

37. Cervantes-Barragan L, Züst R, Weber F, et al (2007) Control of coronavirus infection through plasmacytoid dendritic-cell-derived type I interferon. Blood 109:1131-7. https://doi.org/10.1182/blood-2006-05-023770

38. Scheuplein VA, Seifried J, Malczyk AH, et al (2015) High secretion of interferons by human plasmacytoid dendritic cells upon recognition of Middle East respiratory syndrome coronavirus. $J$ Virol 89:3859-69. https://doi.org/10.1128/JVI.03607-14 
39. Boyette LB, Macedo C, Hadi K, et al (2017) Phenotype, function, and differentiation potential of human monocyte subsets. PLoS One 12:e0176460. https://doi.org/10.1371/journal.pone.0176460

40. Hou W, Gibbs JS, Lu X, et al (2012) Viral infection triggers rapid differentiation of human blood monocytes into dendritic cells. Blood 119:3128-31. https://doi.org/10.1182/blood-2011-09-379479

41. Lambotin M, Raghuraman S, Stoll-Keller F, et al (2010) A look behind closed doors: interaction of persistent viruses with dendritic cells. Nat Rev Microbiol 8:350-60.

https://doi.org/10.1038/nrmicro2332

42. Griffith JW, Sokol CL, Luster AD (2014) Chemokines and chemokine receptors: positioning cells for host defense and immunity. Annu Rev Immunol 32:659-702. https://doi.org/10.1146/annurevimmunol-032713-120145

43. Garg AD, Vandenberk L, Fang S, et al (2017) Pathogen response-like recruitment and activation of neutrophils by sterile immunogenic dying cells drives neutrophil-mediated residual cell killing. Cell Death Differ 24:832-843. https://doi.org/10.1038/cdd.2017.15

44. Chen X, Oppenheim JJ (2014) Th17 cells and Tregs: unlikely allies. J Leukoc Biol 95:723-731. https://doi.org/10.1189/jlb.1213633

45. Wang J, Li Q, Yin Y, et al (2020) Excessive Neutrophils and Neutrophil Extracellular Traps in COVID19. Front Immunol 11:2063. https://doi.org/10.3389/fimmu.2020.02063

46. Cain DW, Cidlowski JA (2017) Immune regulation by glucocorticoids. Nat Rev Immunol 17:233-247. https://doi.org/10.1038/nri.2017.1

47. Galluccio F, Ergonenc T, Garcia Martos A, et al (2020) Treatment algorithm for COVID-19: a multidisciplinary point of view. Clin Rheumatol 39:2077-2084. https://doi.org/10.1007/s10067-02005179-0

48. Menzella F, Biava M, Barbieri C, et al (2020) Pharmacologicaltreatment of COVID-19: lights and shadows. Drugs Context 9:. https://doi.org/10.7573/dic.2020-4-6

49. Park A, Iwasaki A (2020) Type I and Type III Interferons - Induction, Signaling, Evasion, and Application to Combat COVID-19. Cell Host Microbe 27:870-878. https://doi.org/10.1016/j.chom.2020.05.008

50. Channappanavar R, Fehr AR, Vijay R, et al (2016) Dysregulated Type I Interferon and Inflammatory Monocyte-Macrophage Responses Cause Lethal Pneumonia in SARS-CoV-Infected Mice. Cell Host Microbe 19:181-93. https://doi.org/10.1016/j.chom.2016.01.007

\section{Table}

Table 1 - Clinical and demographic characteristics of the included patient cohort 
COVID-19 ICU

patients

$(\mathrm{n}=40$, unless

indicated)

\section{Baseline characteristics}

Age, years (median, IQR)

Gender

Men

Women

Ethnicity

Comorbidities and severity of illness

BMI (median, IQR)

Charlson Comorbidity Index (median, IQR)

SOFA score first 24h upon ICU admission (median, IQR)

Diagnosis of SARS-CoV-2

CT compatible

qRT-PCR nasopharyngeal swab only

qRT-PCR BAL fluid only

qRT-PCR nasopharyngeal swab and BAL fluid

Highest level of respiratory support during ICU stay

Oxygen via oxymask

High flow oxygen support

Invasive \& non-invasive mechanical ventilation

Prone ventilation/inhaled NO/ECMO

$10(25 \%)$

$2(5 \%)$

$11(27 \%)$

$17(42 \%)$

NA

$2[1-3]$

0

$(n=8)$

Caucasian $(\mathrm{n}=39)$

North African $(n=1)$

$3(37 \%)$

$5(63 \%)$

28.3 [24.3-31.3]

[3-8]

0

\section{Immuno-modulatory therapy during ICU stay}

Methylprednisolone only (MP (n))

$19(47 \%)$

$24.0[21.5-26.5]$

Caucasian $(\mathrm{n}=8)$

\begin{tabular}{|lcc|}
\hline Anti-IL6 (n) & $1(2 \%)$ & NA \\
\hline MP + anti-IL1 (n) & $3(7 \%)$ & NA \\
\hline MP + anti-IL6 (n) & $5(12 \%)$ & NA \\
\hline none of the above & $12(30 \%)$ & NA \\
\hline Timeline of blood sampling & & NA \\
\hline Time from illness onset to ICU admission (median, IQR) & $8(5)$ & NA \\
\hline Time from ICU admission to first blood draw (median, IQR) & $\begin{array}{c}1(1) \\
(\mathrm{n}=24)\end{array}$ & \\
\hline
\end{tabular}




\begin{tabular}{|c|c|c|}
\hline $\begin{array}{l}\text { Time from ICU admission to mid-point blood draw (median, } \\
\text { IQR) }\end{array}$ & $\begin{array}{l}12(1) \\
(\mathrm{n}=37)\end{array}$ & NA \\
\hline $\begin{array}{l}\text { Time from ICU admission to blood draw at bronchoscopy } \\
\text { (median, IQR) }\end{array}$ & $\begin{array}{l}15(10) \\
(n=13)\end{array}$ & NA \\
\hline $\begin{array}{l}\text { Time from ICU admission to blood draw at discharge (median, } \\
\text { IQR) }\end{array}$ & $\begin{array}{l}23(16) \\
(\mathrm{n}=38)\end{array}$ & NA \\
\hline \multicolumn{3}{|l|}{ Outcome } \\
\hline Length of hospital stay (median, IQR) & $37(22)$ & NA \\
\hline Length of ICU stay (median, IQR) & $23(21)$ & NA \\
\hline $\begin{array}{l}\text { Length of hospital stay and revalidation after ICU stay (median, } \\
\text { IQR) }\end{array}$ & $\begin{array}{l}24(16) \\
(\mathrm{n}=12)\end{array}$ & NA \\
\hline
\end{tabular}

\section{Figures}



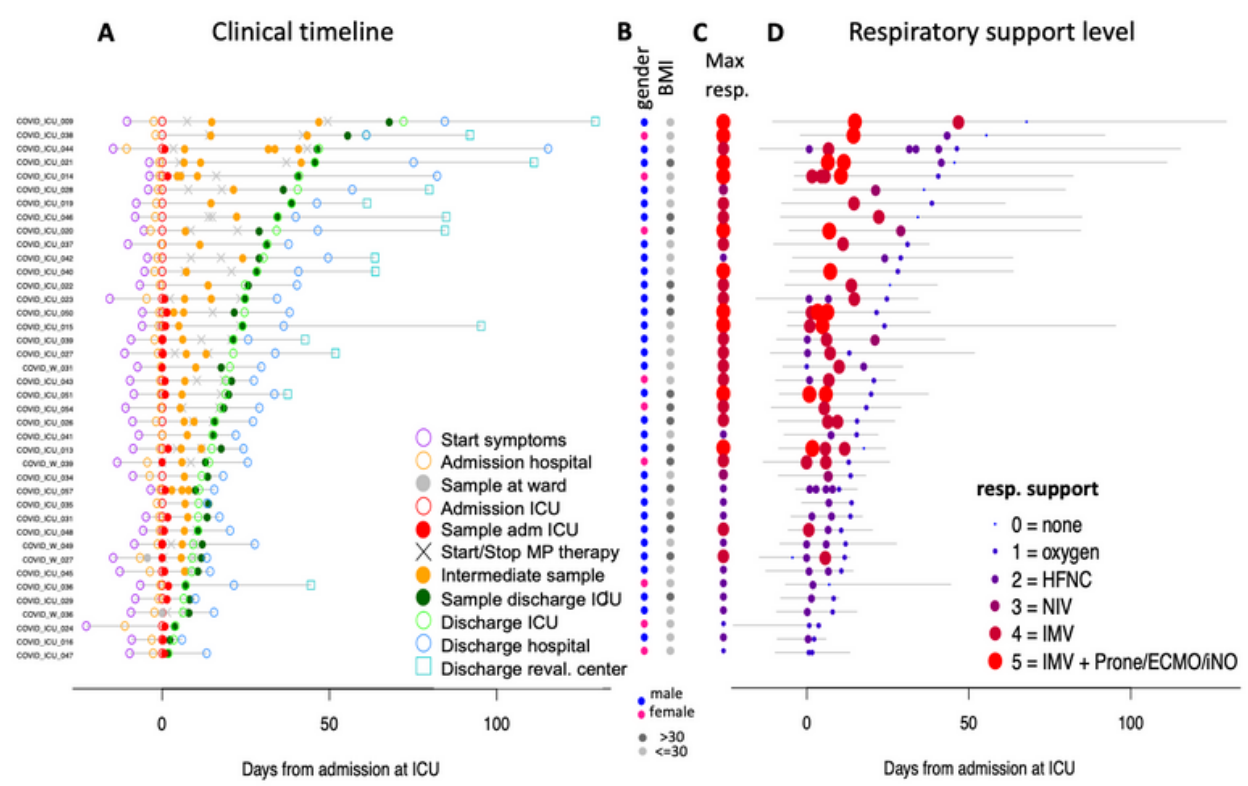

E Blood profiling using CyTOF

F Treatments

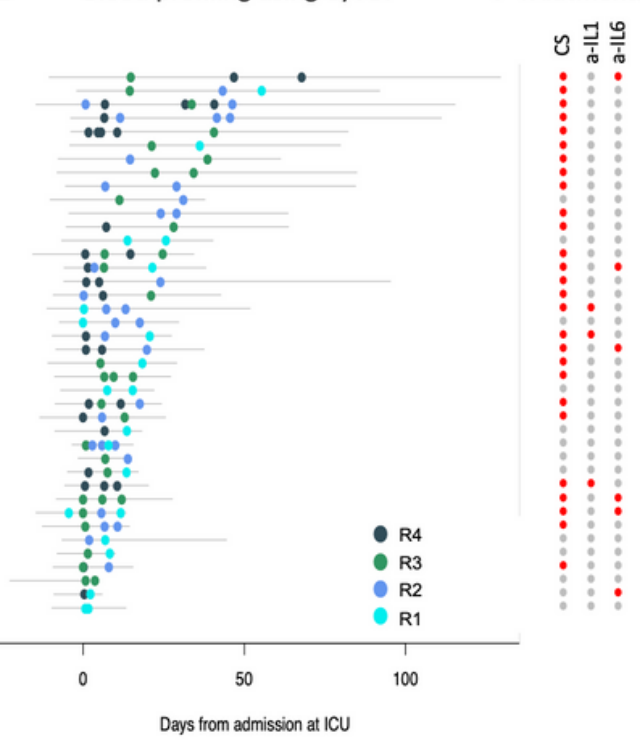

Figure 1

\section{Figure 1}

Detailed timeline of the COVID-19 patient cohort highlighting clinical milestones (A) Line plot on which clinical milestones are highlighted according to their relative time (days) from each patient's admission to ICU. Patients are ordered (top to bottom) according to their overall stay at ICU. (B) Dot plot indicating the gender and $\mathrm{BMI}$ of each included patient. (C,D) Line plot indicating the level of required respiratory support at concomitant sampling times (D) as indicated in panel A. Panel C highlights the maximal level of respiratory support each patient required at concomitant sampling times (C). (E) Mapping of the defined NLR groups (1-4) on top of the indicated timeline as defined in panel A. (F) Indication of the treatments received by each of the patients during their stay at ICU. The color and symbol code are indicated in each separate panel. MP: methylprednisolone, a-IL1: anti-IL-1, a-IL6: anti-IL6, HFNC: high flow nasal cannula, NIV: non-invasive ventilation, IMV: invasive mechanical ventilation, ECMO: extracorporeal membrane oxygenation, iNO: inhaled nitric oxide, BMI: body mass index 


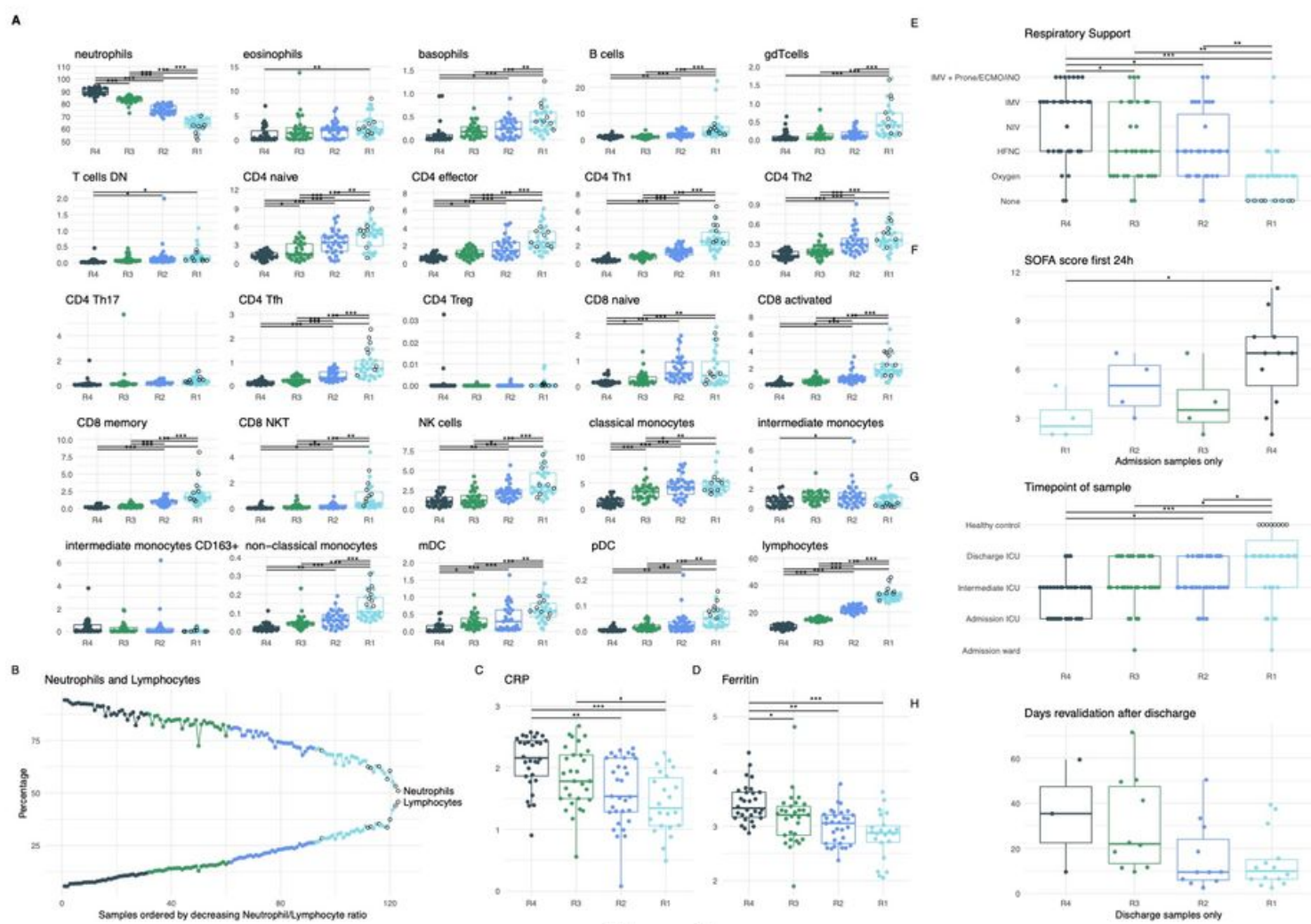

Figure 2

\section{Figure 2}

Longitudinal Immunoprofiling of Peripheral Blood Profiles and Integration with Clinical Features (A) Overview of immune cell population percentages, grouped by NLR, where R4 indicates the most severe neutrophilia/lymphopenia and R1 shows similar values to healthy controls (HC). (B) Neutrophil and lymphocyte percentages, ordered by decreasing NLR. $(C, D)$ Levels of acute phase reactants, C-reactive protein and ferritin, across all ratio groups. (E) Distribution of respiratory support levels and (F) SOFA score of the first $24 \mathrm{~h}$ upon admission to ICU over the NLR groups. (G) Longitudinal sampling time points of patients from admission into Ward and ICU, mid-stay and discharge as grouped by NLR. (H) Days of revalidation upon discharge from ICU as grouped by NLR. (A-H) Color indicates NLR group: black: R4, green: R3, blue: R2, cyan R1. HC are indicated as black open circles. ${ }^{\star \star \star} p \leq 0.001,{ }^{\star \star} p \leq 0.01,{ }^{*} p \leq 0.05$ 


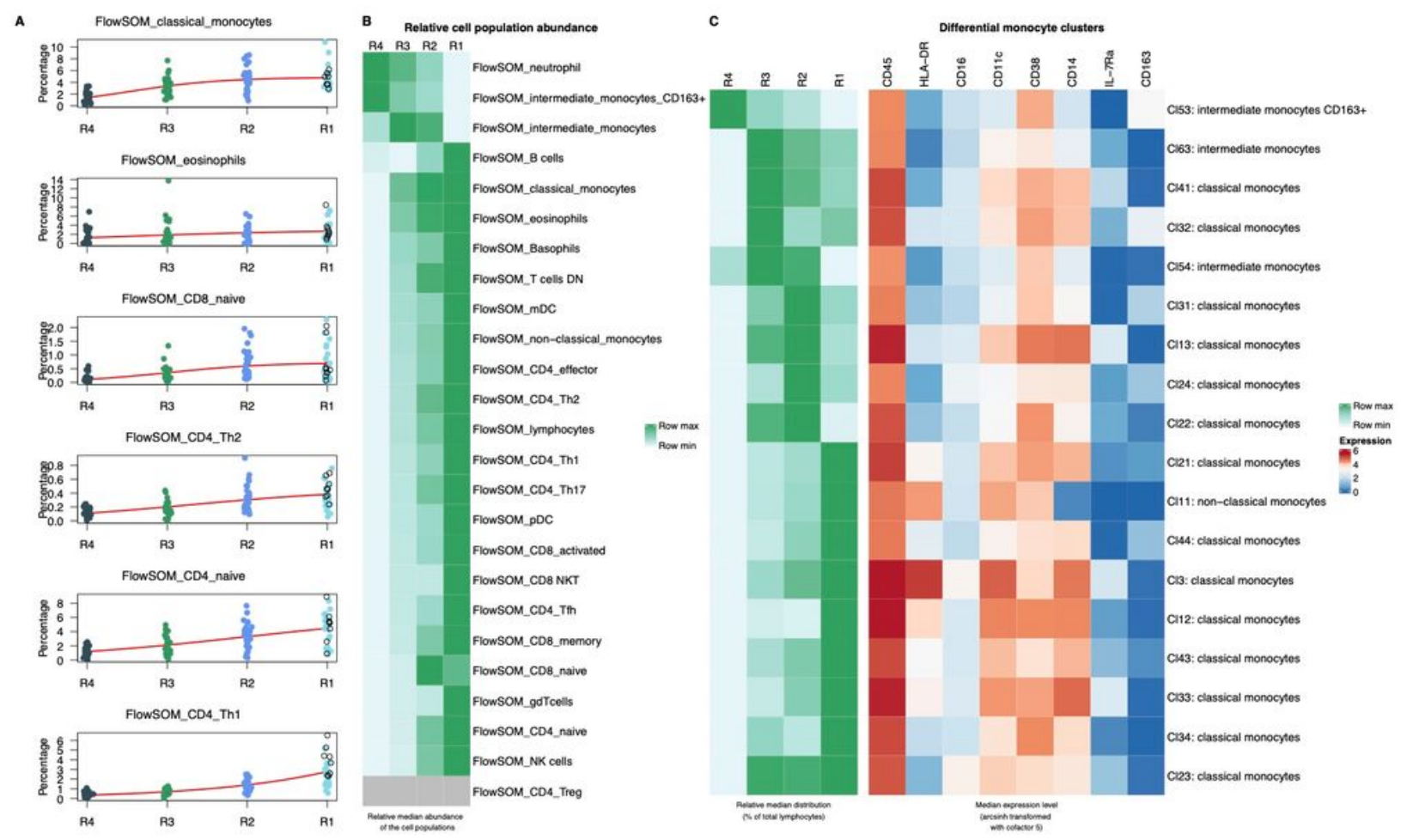

Figure 3

\section{Figure 3}

Overview of Cell Populations Abundances in Peripheral Blood Profiles (A) Abundance of selected populations shown, fitted with a dose response curve using a three-parameter logistic model (red line). Individual samples colored by NLR, black open circles for healthy controls (HC). (B) Median abundance per NLR group. Values are scaled per row, dark green indicating in which group the cell population is the most abundant. CD4_Tregs had a median of 0 in all profiles, indicated in grey. (C) Overview of all FlowSOM clusters labelled as monocytes. On the left, the relative abundance per NLR group (similar to B), on the right the median marker expression for those clusters. 


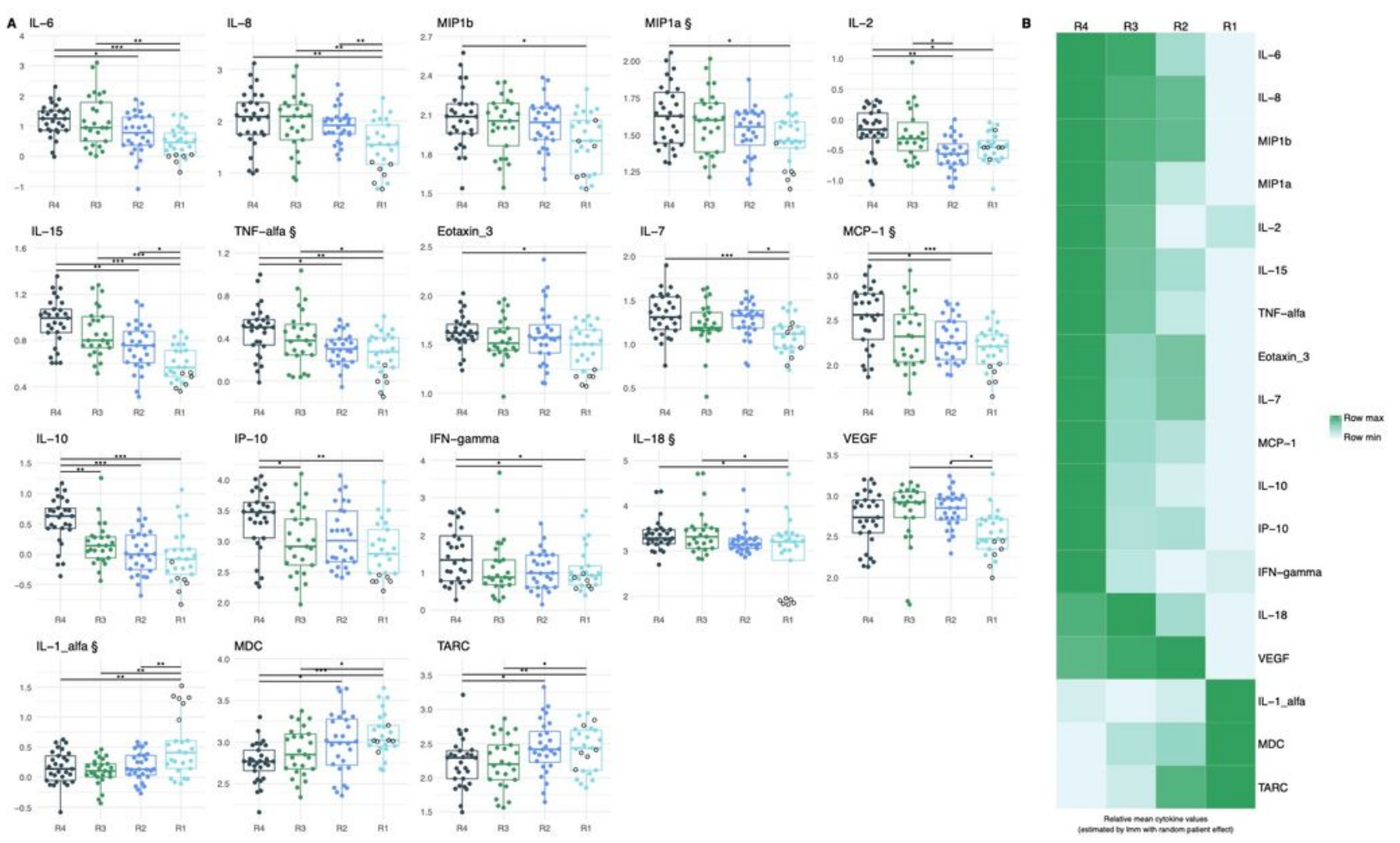

Figure 4

\section{Figure 4}

Cytokine and chemokine profiling according to the Peripheral Blood Profiles Serum levels of 32 proinflammatory soluble proteins were determined by multiplexed ELISA assay concurrent with the whole blood sampling times. (A) Box plots illustrate the correlation of the level of the significantly different cytokine/chemokine with the outlined R1-4 (log10 scale, filled circle presents a COVID-19 patient, black empty circle illustrates a healthy control patient). (B) Heatmap generated by clustering analysis of the cytokine expression signatures across R1-4. ${ }^{* \star} \mathrm{p} \leq 0.001,{ }^{* *} \mathrm{p} \leq 0.01,{ }^{*} \mathrm{p} \leq 0.05$ 
A

C
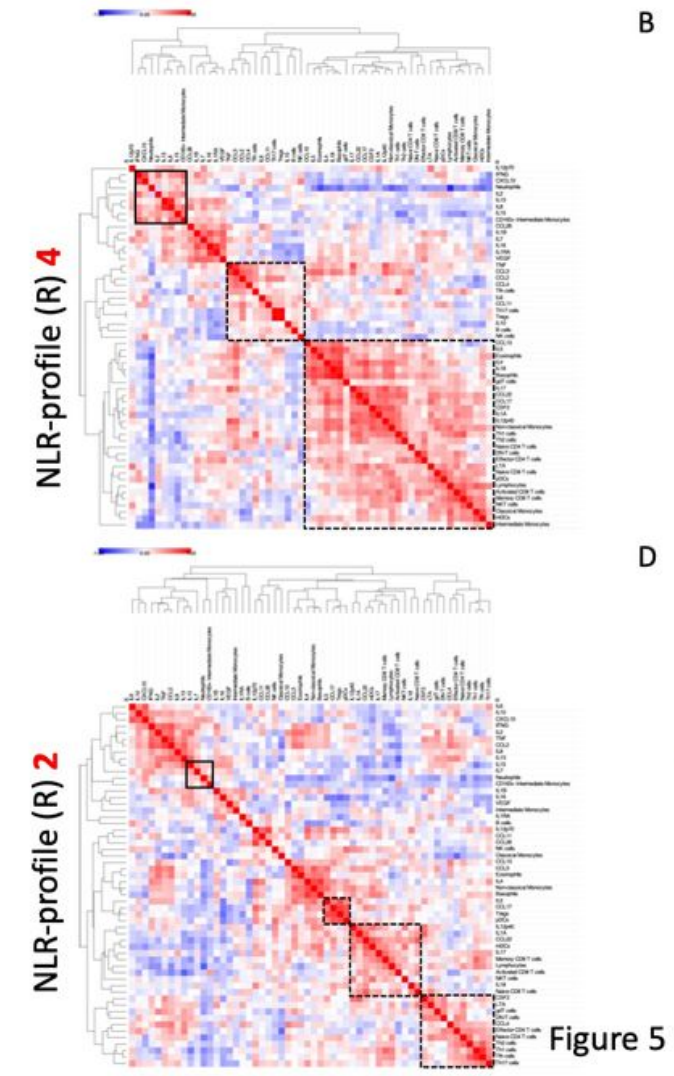

B

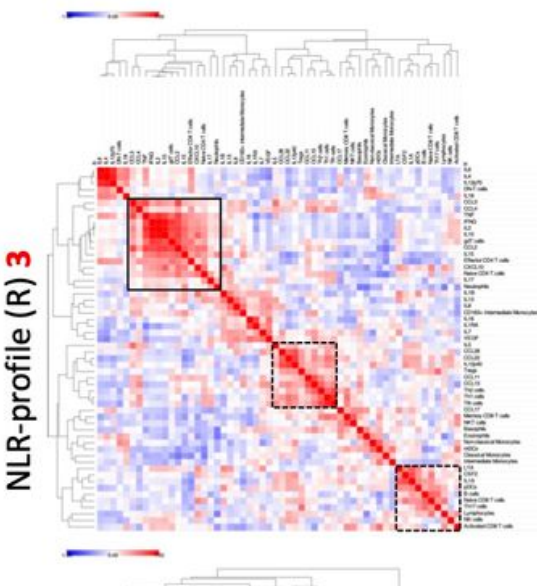

D

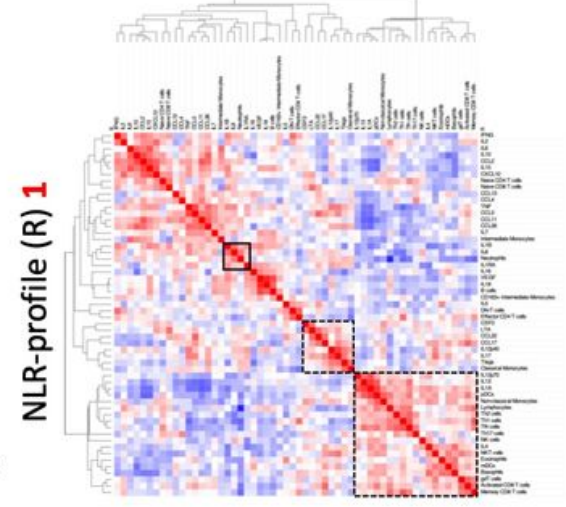

Figure 5

Integration analysis of the blood profiles and cytokine levels Pearson correlation-driven similarity/correlation matrix analysis of cytokines/chemokines and mass cytometry data across the different COVID-19 patient's NLR subgroups i.e., R4 (A), R3 (B), R2 (C) and R1 (D). Herein, this matrix is a statistical modelling representation wherein statistically stable correlations between variables across different patients, facilitates their co-clustering as indicated by the dendrograms. The diagonal correlation value is 1, which denotes the highest possible statistically-significant correlation value that is "centering" our correlation matrices.

\section{Supplementary Files}

This is a list of supplementary files associated with this preprint. Click to download.

- SupplementaryTables.docx

- Suppl.Table3.xIsx

- suppfig1.jpg

- suppfig2.jpg

- suppfig3.jpg

- suppfig4.jpg

- suppfig5A.jpg 
- suppfig5B.jpg

Page 25/25 